\title{
Joint Penetration Monitoring in Low-Frequency Pulsed GTA Root Pass Welding of Medium-Thick Steel Plates
}

\author{
Low frequency and large duty-cycle pulsed GTAW was employed to weld the \\ medium-thick steel plates with a 5-mm root face and 2-mm root opening
}

BY Z. ZHU, G. ZHANG, K. WANG, Y. SHI, AND M. ZHU

\begin{abstract}
Welder-dependent manufacturing is no longer suitable for the modern production of a high-performance nuclear pressure container. The high-quality root pass welding of medium-thick steel plates is the main challenge to obtain a sturdy reactor vessel, especially to generate one-sided welding with back-formation bead without a backing. Herein, low frequency and large duty-cycle pulsed gas tungsten arc welding (GTAW) was employed to weld the medium-thick steel plates with a 5-mm root face and $2-\mathrm{mm}$ root opening. The arc characteristic and weld pool dynamic behavior in the proposed GTA root pass welding was investigated by a highspeed camera, and a deflection phenomenon of arc tail flame was first found. The correlations of the characteristic parameters of the arc tail flame, including the deflected angle and length, with the weld joint penetration and welding speed were also analyzed in detail. The results showed a negative correlation to the welding speed and a positive correlation with the weld joint penetration. A sound weld bead was formed at a range from $15 \mathrm{deg}$ and $20 \mathrm{~mm}$ to 19 deg and 27 $\mathrm{mm}$. Based on the above relationship, a new method using these two characteristic parameters was proposed to identify the weld joint penetration in the root pass welding, and its fundamentals were completely demonstrated by the dynamic change of the keyhole. Its feasibility was also demonstrated by the experiment combined with the weld pool dynamicdependent theoretical analysis.
\end{abstract}

\section{KEYWORDS}

- GTAW • Weld Joint Penetration • Root Pass Welding

- Arc Tail Flame • Medium-Thick Steel Plates

\section{Introduction}

Gas tungsten arc welding (GTAW) is an essential fusion welding process in the fabrication of complex, heavy thick steel plate-based structures, such as nuclear pressure vessels and engineering machinery, due to its high/reliable welding quality (Refs. 1, 2). Particularly, manual GTAW is still widely adopted in the root pass welding of medium-thick steel plates under variable conditions because skilled welders can assure a desired high weld quality by adjusting the welding parameters (e.g., welding torch orientation, welding speed, or arc length) via observations of the molten pool surface. However, the welding efficiency is highly dependent on the welders' responses, and the weld quality of nuclear pressure vessels cannot be guaranteed due to tired human operations, unstable welding skills, or other influences.

With the rapid development of the advanced nuclearpower industry, the number of nuclear pressure vessels used to enclose the spent fuel or nuclear waste have been exponentially increased (Ref. 3). In addition, the weld procedure and quality level of nuclear pressure vessels have changed in accordance with the new version of the Guidebook for the Design of ASME Section IX Pressure Vessels (Ref. 4). In this guide, the root pass welding of the pressure vessel is only allowed without backing against the corrosion cracks or incomplete fusion. Moreover, the deeper root pass weld joint penetration is beneficial to decreasing the filler metal of the weld groove and improving the welding efficiency. To this end, the development of a high-efficient/ qualified, reliable, and automatic welding method is very important for the production of a good weld bead with one-side welding with back formation for thick-plate-based welded structures.

In recent years, a modified GTAW named K-TIG (keyhole tungsten inert gas)/K-GTAW was developed and was applied to weld the medium-thick steel plates. K-GTAW is defined by ASME as one of many variants of the GTAW process, where a "keyhole" is initiated and the molten pool solidifies and closes the keyhole behind the direction of torch travel by surface tension forces (Ref. 5). To generate a keyhole, two elements are necessary. One is a higher welding current (> $300 \mathrm{~A}$ ) to pro- 

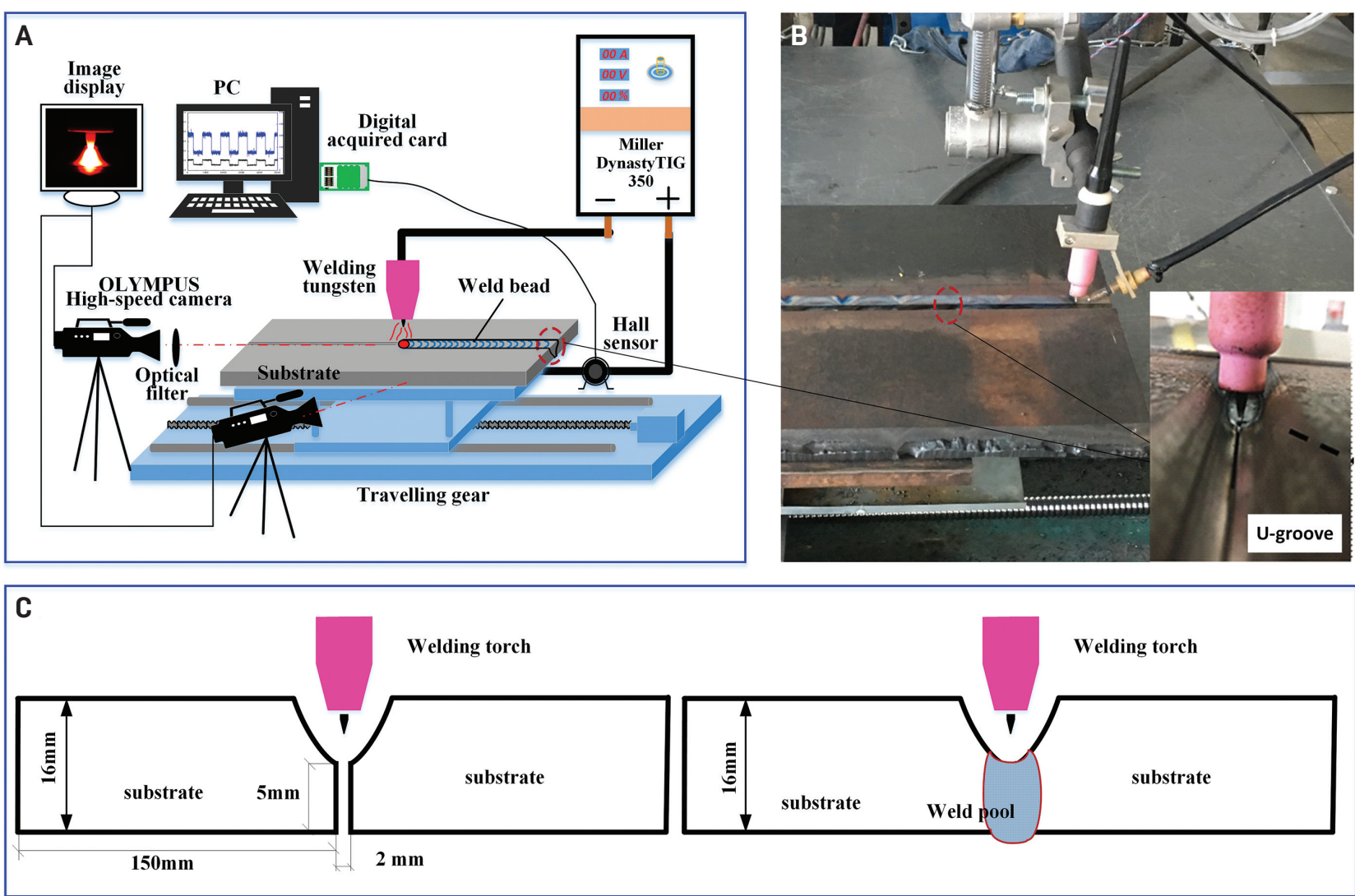

Fig. 1 - Schematics showing experiment system and sample setup: A - Welding system diagram; B - GTAW system; C - sample setup and groove type.

duce a higher arc pressure to displace the liquid metal to dig into the liquid weld pool to form a keyhole. Another is a high cooling system for the tungsten electrode when the electrons are emitted from a much smaller region at its tip. Thereby, the structure and volume of the welding torch is more complex and larger than that of conventional GTAW. In addition, the mandatory need for high current makes its application range be narrowed when a good material property is required (Refs. $6-8)$. In actual productions, a complete and consistent weld joint penetration of the K-TIG/K-GTAW process was obtained and was maintained by strictly controlling the welding current and other welding parameters. The closed-loop control of weld joint penetration may not be easy to be realized (Refs. 9, 10).

Reliable and real-time monitoring of the weld joint penetration is the main challenge of automatic one-sided welding with back formation and deeper penetration methods. Sensing and control technologies for weld joint penetration may include machine vision (Refs. 11-14), radiography (Ref. 15), infrared sensing (Refs. 16-18), ultrasonic (Refs. 19-21), magnetic detection (Ref. 22), acoustic measurements (Refs. 23, 24), and weld pool oscillation (Refs. 25-28). Chen et al. (Refs. 29, 30) set up a computer with a passive vision system to acquire the key features related to the 3D weld pool surface, and established a supervised machine model to predict the weld pool backside width. However, this method cannot be employed in large welding current conditions due to the strong intensity of the arc light. Although ultrasonic-based measurement lacked visual images and employed highly skilled humans to conduct weld penetration detection, it has been widely applied due to its flexibility and low cost (Ref. 20). Chandrasekhar et al. (Ref. 31) reported a new means of using hybrid soft computing techniques to accurately estimate the weld bead width and penetration depth from the infrared radiation (IR) thermal image of the weld pool, and extracted four features from the hot spot of the IR thermal images. An artificial neural networkbased model was further built, in which the welding current and the four features of the IR thermal images were used as inputs, and the measured bead width and penetration depth were chosen as the outputs. The experimental results indicated a good correlation between the measurements and estimations. However, the temperature gradient was extremely high within the weld pool and its surrounding area. To realize the measurement to derive the weld penetration, the measured point must be accurately positioned. Indeed, an error can be expected under varying conditions. Pal et al. (Ref. 23) studied the relationship between the acoustic emissions and joint penetration by using the amplitude and centroid frequency of the acoustic emission. This approach requires noise reduction and an efficient data processing system when the welding penetration statuses are changed. In recent years, an alternative arc voltage-based method was proposed to identify the weld penetration, and a step-by-step pulsed GTAW complete joint penetration was achieved (Refs. 32, 33).

The above-mentioned investigations have demonstrated 


\begin{tabular}{|c|c|}
\hline Parameters & Value \\
\hline Peak current $I_{p} / \mathrm{A}$ & 310,320 \\
\hline Base current $I_{b} / A$ & 62,64 \\
\hline Duty cycle $D / \%$ & 80 \\
\hline Welding speed $\mathrm{V} / \mathrm{mm} / \mathrm{min}$ & $60 \sim 110$ \\
\hline Distance from tungsten tip to root face $\mathrm{H} / \mathrm{mm}$ & 2 \\
\hline Tungsten diameter $\mathrm{d} / \mathrm{mm}$ & 3.2 \\
\hline Flow rate of shielding gas (Ar) $f / \mathrm{L} \mathrm{min}^{-1}$ & 10 \\
\hline
\end{tabular}

that some characteristic features of the weld pool were closely correlated with weld joint penetration and have been used to realize the feedback control of weld penetration through neural network or transfer function modeling. Many studies have examined the weld pool oscillation method in these developed approaches, to which the oscillation frequency of the weld pool without metal transfer was generally employed to estimate weld penetration (Refs. 34-37). A direct physical correlation between the weld pool oscillation and weld joint penetration can be established from a physical welding perspective. As such, measurement and control of the weld pool oscillation can achieve a complete weld joint penetration bead. Unfortunately, this methodology was always applied to sense and control the plates jointed without an opening and groove. The generation of a reliable oscillation frequency signal during the whole welding process with a varying root opening may not be achieved in the in-situ application of nuclear pressure vessels or other thick plate pipes, due to welding conditions changes, such as 1 to $2 \mathrm{~mm}$ root opening and V-/U-groove. To this end, our previous studies (Refs. 38-40) found that the weld penetration status enables precision control by monitoring the weld pool oscillation frequency and adjusting the welding speed. Furthermore, by observing the dynamic variation of the weld pool, we found that the weld metal fluid flow in the root opening condition was more intensive and confusing than that without the root opening. As a result, a variable frequency signal that did not accurately estimate the weld penetration status and an ununiform weld penetration bead was obtained.

As previously stated, the welding process of mediumthick steel plates currently consists of two in-situ manufacturing procedures, including root pass welding and fillingcover welding with a submerged arc welding method. A large root face of groove is generally utilized to avoid burning through the root pass weld bead when the filling-cover welding is operated. This also enhances the welding efficiency by melting more base material. In this work, a low frequency with a large duty-cycle pulsed GTAW process was employed to weld the root pass of medium-thick steel plates with a 2 $\mathrm{mm}$ root opening. Compared with the K-TIG and the traditional GTAW, using the large duty-cycle pulsed current not only melted more base metal and increased the weld pool depth at long-term peak current but also enhanced the strong arc force to pierce the weld pool to generate a key- hole. Furthermore, the low frequency of the pulsed current was adopted to stir and to decrease the intensity of the weld metal fluid flow for keeping the stabilization of the keyhole evolution. The arc characteristic and weld pool dynamics were first investigated in the GTA root pass welding process with a low-frequency pulsed current. The effects on the weld joint penetration of the U-groove medium-thick plates with a root opening and the one-sided with back-formation weld bead were also experimentally studied. The correlations of the deflected angle and the length of the arc tail flame with the weld joint penetration were further analyzed in detail. Simultaneously, a new means of using these two parameters was proposed to estimate the weld joint penetration, and its feasibility was demonstrated by the experiments. The remainder of this paper is organized as follows: experimental setup, results and discussion, and the conclusion.

\section{Experimental Setup}

The butt joint with U-groove was adopted, and the pulsed GTA root pass welds without filler metal were performed on a $500 \times 150 \times 16 \mathrm{~mm}$ dimensional $16 \mathrm{MnR}$ steel substrate that is widely used to produce the nuclear pressure container due to its favorable mechanical and welding properties. Before starting the welding, the root pass of the U-groove was completely polished by sanding and was cleared with alcohol to remove rust, oil stain, moisture, and other impurities. Both ends of the two workpieces were first welded. The shielding gas was pure argon. The electrode was ground to the flat tapered shape, and its angle was about $20 \mathrm{deg}$. The inner diameter and length of the cup was 8 and $47 \mathrm{~mm}$, respectively. The schematic diagrams of the experimental system and groove shape are shown in Fig. 1.

Figure 1 shows the low frequency and large duty-cycle pulsed GTAW experiment system as well as the sample welded setup, which includes three parts: the visual sensing system, pulsed current welding system, and movement control system. The welding and sensing system comprises a Miller Dynasty ${ }^{\circledR}$ TIG 350 power source, an Olympus I-speed 3 camera, a set of travelling gear and controller, and a composite filter lens that consists of a neutral filter and a band pass filter. The band pass filter is a 1-in.-(25.4-mm-) diameter filter with a center wavelength of 660/1064 $\pm 10 \mathrm{~nm}$. A highspeed camera that can take 60 to 5000 frames s$^{-1}$ was used to capture the arc and weld pool surface images. The back- 

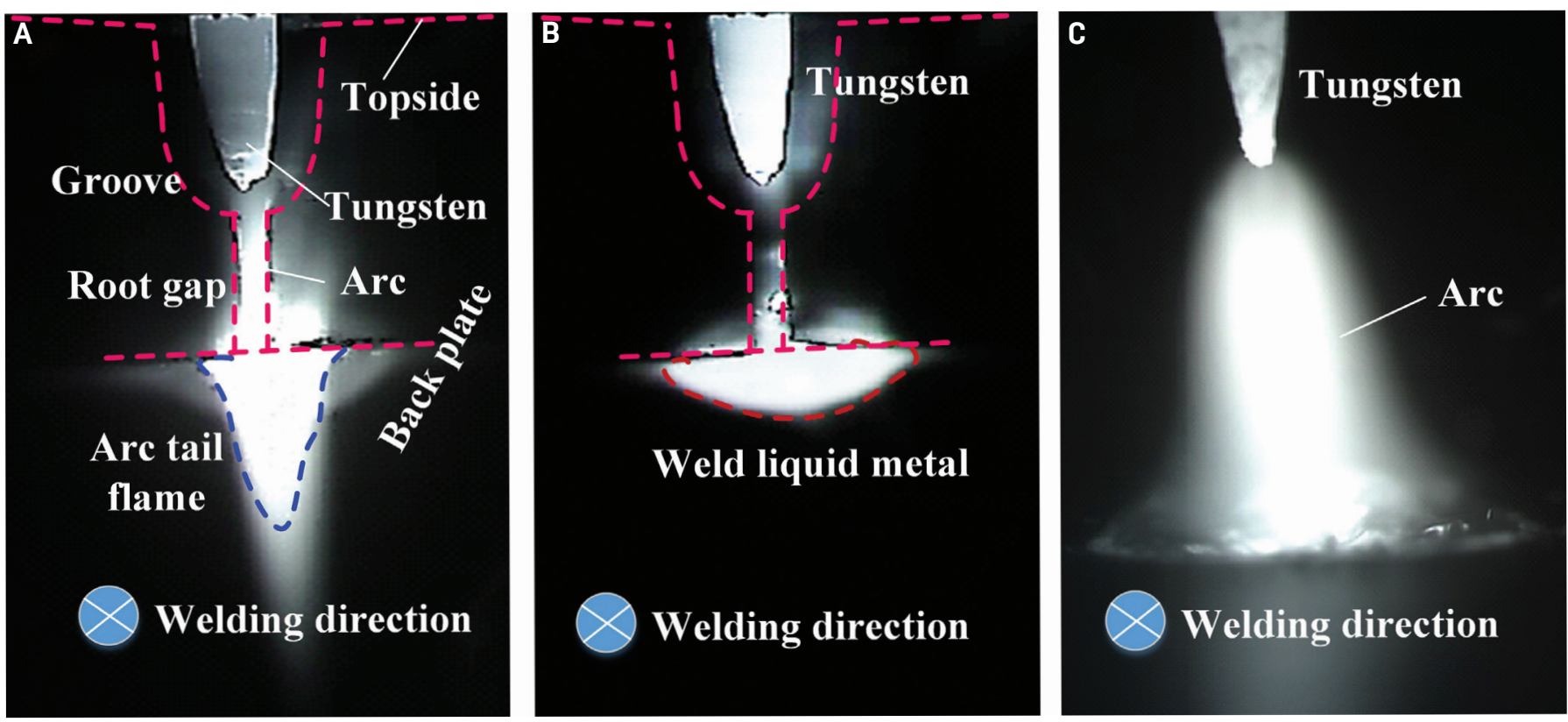

Fig. 2 - The selected typical arc shape images: $A$ - Peak current period; B - base current period; C - normal arc shape with direct current.

side camera was almost positioned parallel with the bottom of the workpiece. Being fixed perpendicularly to the substrate, the GTAW torch was stationary, whereas the workpiece is traversed below. To diminish the influence of strong arc light further, the high-speed camera was fitted with a composite filter lens. Thus, most of the arc radiation interference, which was distributed along the entire visible wavelength spectrum, was eliminated. The experimental parameters are shown in Table 1.

\section{Results and Discussion}

\section{Analysis of Arc Characteristics}

As we know, the arc shape and its dynamic behavior strongly affect the three dimensions, weld pool surface deformation, and weld joint penetration. To carefully investigate the dynamic change of the arc shape in U-groove root pass welding with a low frequency and large duty-cycle pulsed current action, a series of experiments utilizing the welding parameters in Table 1 were performed. The continuous arc morphologies with varying penetration were also sampled in real-time using a highspeed camera. Specifically, the change of arc dynamic in the peak and base current period was further observed and analyzed in detail. Some of the typical images of arc shape captured at different times were selected and are shown in Fig. 2, which were recorded along the welding direction.

As compared with the bell-like normal arc shape of autogenous GTAW shown in Fig. 2C, the arc in the peak current period shown in Fig. 2A was obviously compressed by two sides of the root face. As a result, a higher-energy density that could melt more root face metal and form a larger volumetric weld pool was generated. As the welding time acted by the peak current increased, the weld joint penetration further increased. In addition, the compressed arc pierced the weld pool, generating a keyhole when the surface ten- sion of the weld pool could not compensate the mutual action of gravity, arc pressure, and gas pressure. The back-arc shape followed a conical morphology, which was named the arc tail flame. At that moment, the root face metal was completely fused, forming a complete joint penetrated weld pool. However, during the base current period, the arc current density and pressure decreased, as shown in Fig. 2B, and the arc heat input into the weld pool was not enough to melt the bottom metal in the root face and only maintained the liquid status of the weld metal. Moreover, the decreased arc pressure was unable to generate the keyhole. Liquid metal from the front of the weld pool filled the keyhole during the base current time, and later flowed backward due to surface tension and electromagnetic force. That is to say, the proposed pulsed welding process achieves keyhole conditions during the peak current, while nonkeyhole complete joint penetration is maintained during the background current.

To deeply study the forming mechanism of a keyhole in the root pass welding, the change of arc dynamic and weld pool behavior from the base period to the peak period was observed and analyzed in detail. The captured images are shown in Fig. 3.

According to Fig. 3, the generating process of the arc tail flame may include the following three stages: 1) arc compression and root face melting; 2) weld pool penetrated and thin film formation; and 3) thin film of the weld pool bottom piercing and keyhole generation. In the first stage, as the peak current was employed at the end of the base current, the heat input into the weld pool and the temperature of the liquid weld metal simultaneously increased, resulting in the lower surface tension of the weld pool and larger gravity due to more substrate metal melting, which was demonstrated by Meng et al. (Ref. 41). During the second stage, the continuous action of the large pulsed peak current significantly enhanced the plasma arc pressure on the weld 


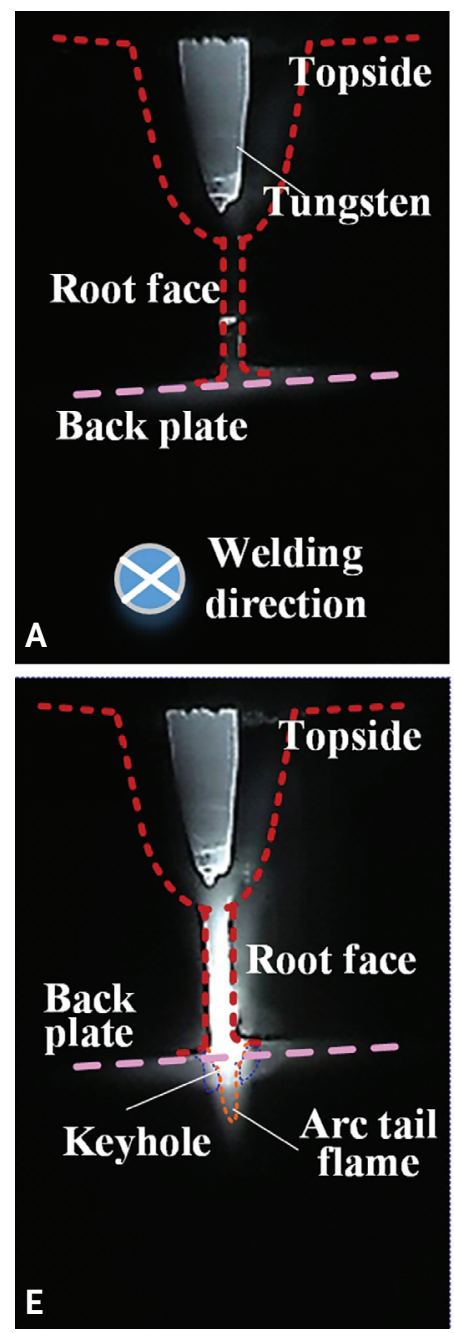

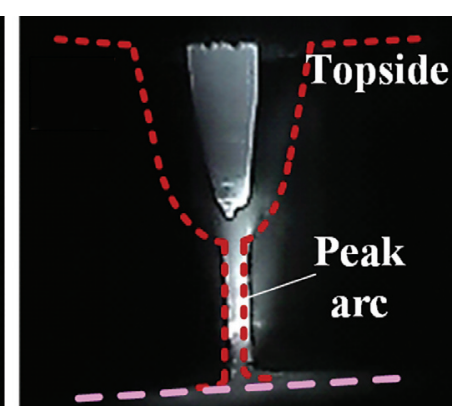

Back plate
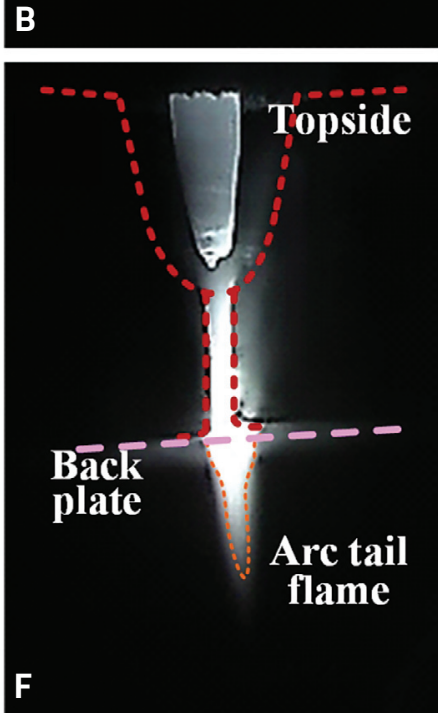

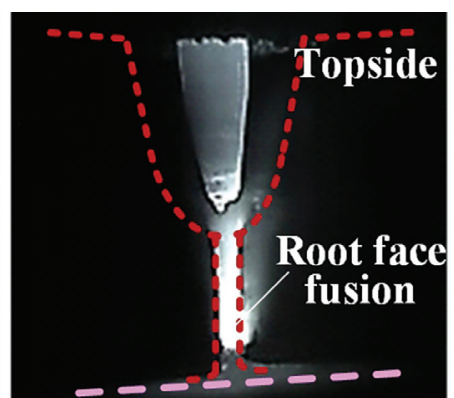

Back plate
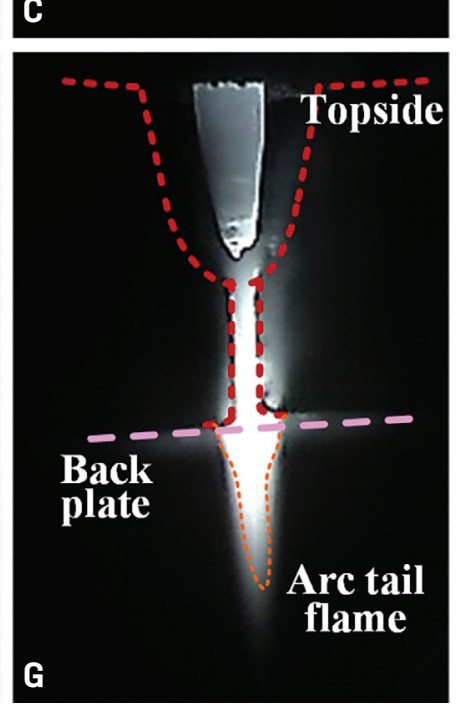
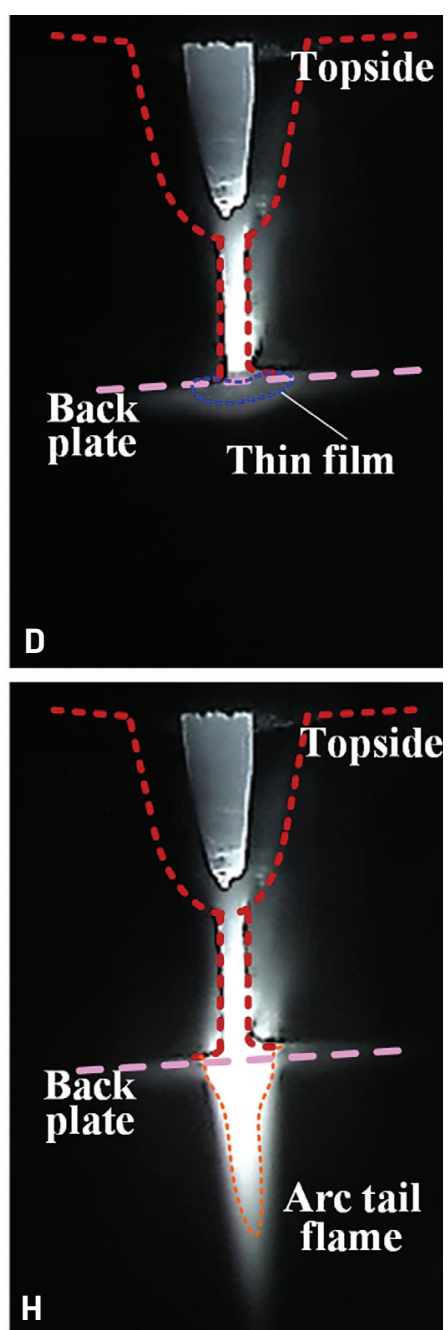

Fig. 3-Arc shape captured from the base current to the peak current period (interval time $20 \mathrm{~ms}$ ).

pool surface, such that the melted metal was easily pushed to the tail of the weld pool with a traveling workpiece.

Hence, the weld pool was completely penetrated, and its bottom under the center of the arc column changed and formed a thin film. In the third stage, the gravity of the remaining melted metal overcame the surface tension under the plasma arc force, and pierced the thin film of the weld pool, thus generating the keyhole. Based on the above analysis, keyhole formation was primarily inferred given that the melted metal in the weld pool under the center of the arc column was squeezed out and pushed to the tail of the weld pool, generating a thinner weld pool bottom film. Thereby, the welding method employed in this work is able to increase the weld joint penetration through imposing a large plasma arc force on the weld pool surface with the large duty-cycle pulsed current, which provides a possible implementation path for achieving a complete joint penetration weld bead formation in thick-medium steel plates welding.

\section{Deflection Phenomenon of the Arc Tail Flame}

A dynamic change of arc morphology along the perpendicular in the welding direction was observed in the above section, and its characteristic was analyzed at different times. At this point, the arc tail flame images were captured by a high-speed camera. To reduce the influence of the strong arc light and clearly observe the change of the arc tail flame with varying joint penetration, a band-pass filter with a center wavelength of $660 \pm 10 \mathrm{~nm}$ was installed in the front of the camera. The high-speed camera was positioned on the same plane with the back plane of the workpiece, with a sample orientation that was placed perpendicular to the welding direction (Fig. 1). The images of arc tail flame captured in the peak current time are shown in Fig. 4.

From Fig. 4, it is observed that the arc tail flame visibly deflected along the welding direction, and its length changed with the different weld penetrations. Figure $4 \mathrm{~A}$ and $\mathrm{C}$ combined with Fig. 3B and D show that the root face was largely melted following an increase in the heat input of the weld pool at the large duty-cycle peak current period. When the mass of the melted metal in the root face achieved a critical value where the surface tension balanced against the arc pressure and gravity of weld pool, the welding arc burned through the weld pool, thus forming a keyhole and generating an arc tail flame on the back of the weld pool. Furthermore, if the pulsed current parameters were matched well with the welding speed, the welding process stabilized and the morphologies of arc tail flame changed less with the variable evolution of keyhole, as 


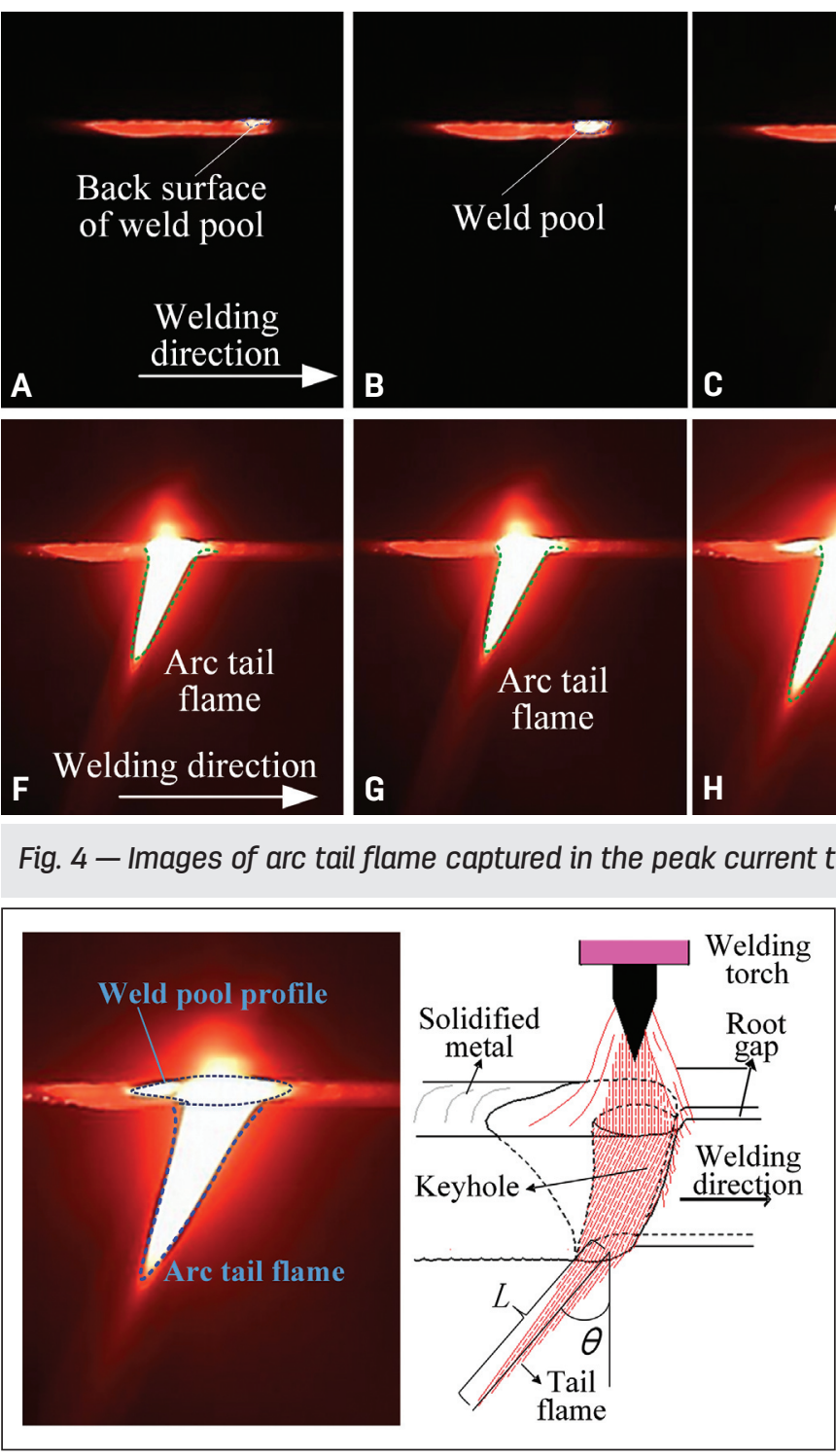

Fig. 5- Schematic model of keyhole and arc tail flame.

shown in Fig. 4E and H. According to the arc tail flame analysis in the different parameterized experiments, the jetting orientation of the arc tail flame was not perpendicular to the welding direction and deflected opposite to the welding direction relative to the torch frame of reference, which was first defined as the deflected angle $\theta-$ Fig. 5.

To further demonstrate the special characteristic of the arc tail flame, many repeatable experiments were performed using the Table 1 parameters. The experimental results showed when the length $L$ and the deflected angle $\theta$ of the arc tail flame fluctuated in a small range through substrate movement, sound one-sided welding with the back-formation bead would be produced. By analyzing the varying process of the arc tail flame, the heat input into the base metal per time in the peak current period may have minimally changed with a constant welding speed. As a result, the dimensions of the weld pool stabilized, and the mutual force acted on the weld pool varied slightly, thereby generating and continuously transferring a relative fixed dimensional keyhole along with the welding movement direction.
Comparisons of the weld pool achieved in the stable and unstable welding processes further showed that the dimensional changes of the keyhole were strongly dependent upon the length and deflected angle of the arc tail flame, which in turn greatly determined the joint penetration and weld bead formation. Consequently, the distinct arc tail flame phenomenon suggests the possible monitoring and identification of the weld joint penetration in the root pass welding of medium-thick steel plates using the length and deflected angle of the arc tail flame.

\section{Correlation of the Arc Tail Flame with the Weld Joint Penetration}

To demonstrate the assumption of using the length and deflected angle of the arc tail flame to estimate the weld joint penetration, the Table 1 parameters were experimentally performed on the given scaled $16 \mathrm{MnR}$ substrates. The images of the arc tail flame under different welding speeds were captured by the Olympus I-speed 3 camera with a sampling frequency pre-set to $1000 \mathrm{~Hz}$. Moreover, the length and deflected angle of the arc tail flame were measured by the integrated software of the OLYMPUS I-speed camera -Fig. 6. Based on the measured data, the qualitative relationship of the four characteristic arguments, including the length, deflected angle, welding speed, and weld joint penetration, was statistically curved and shown in Fig. 7.

Figure 7 presents the changeable trends of the length and deflected angle of the arc tail flame at varying welding speeds, and the weld bead formation was finally predicted. In general, when the welding speed increased, the length and deflected angle of the arc tail flame decreased, and the weld joint penetration gradually changed from burnthrough, collapse status, complete joint penetration to incomplete fusion status. In addition, a length and deflected angle range of 10 to $30 \mathrm{~mm}$ and 15 to $25 \mathrm{deg}$, respectively, generated a dynamically stabilized keyhole, thus resulting in 

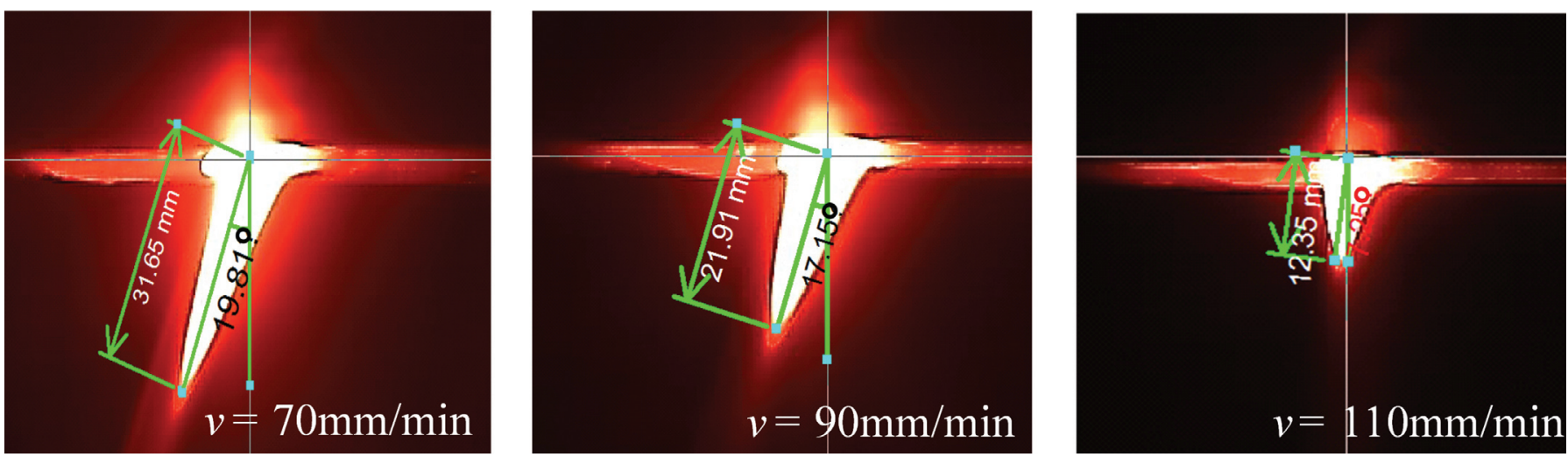

Fig. 6 - Measured length and angle with different welding speed.

a uniform, complete joint penetration weld.

The physics of the welding fundamentals-based analysis indicated that the heat input into the base metal decreased with increasing welding speed, resulting in a smaller dimensional keyhole. As the welding speed further increased, the heat input into the weld pool insufficiently melted the bottom of the weld pool. As a result, the necessary thin film generation of the keyhole was very difficult, given that the weld pool was not complete joint penetrated, and an incomplete fusion weld bead was achieved. This fusion status was presented on the changes of the arc tail flame characteristics, including the decreases in length and deflected angle. On the contrary, slower welding speeds generated greater energy inputs and formed a larger keyhole, which made the most of the burning arc pierce of the keyhole, thus producing a special arc tail flame with a longer and greater deflected angle. On the other hand, the slower welding speed benefited in the displacement of the weld pool melted metal to the weld pool sides or tail due to the arc force and gas pressure, which ultimately improved the fluid mobility of the weld metal. Consequently, higher temperature liquid metal transferred to the bottom of the weld pool and enhanced root face metal melting, which easily formed the thin film and the keyhole. Thereby, based on the above analysis, the length and deflected angle of the arc tail flame were used to estimate the status of the weld joint penetration and predict the weld quality of one-sided welding with backformation bead.

The above analysis and assumptions were further verified by the following experiments. In these experiments, the welding speed was chosen to be $90 \mathrm{~mm} / \mathrm{min}$. The peak and base current was set to 320 and $64 \mathrm{~A}$, respectively. Pulsed frequency and duty-cycle were $1.5 \mathrm{~Hz}$ and $80 \%$, respectively. The sampling frequency of the high-speed camera was 1000 $\mathrm{Hz}$. The typical images of the arc tail flame selected in a whole pulsed current period are shown in Fig. 8. The selected interval time in the base current and peak current period was 100 and $47 \mathrm{~ms}$, respectively. The weld bead formation and the x-ray image are shown in Fig. 8.

As shown in Fig. 8A, the arc tail flame was stabilized and maintained in a whole peak current period, and its deflected angle and length changed slightly. After being measured by software, the results displayed that the deflected angle ranged from 15 to $19 \mathrm{deg}$, and the length was in the range of 20 to 7 $\mathrm{mm}$. Figure $8 \mathrm{~B}$ shows complete joint penetration without any surface defect in the U-groove root pass weld bead. Moreover,

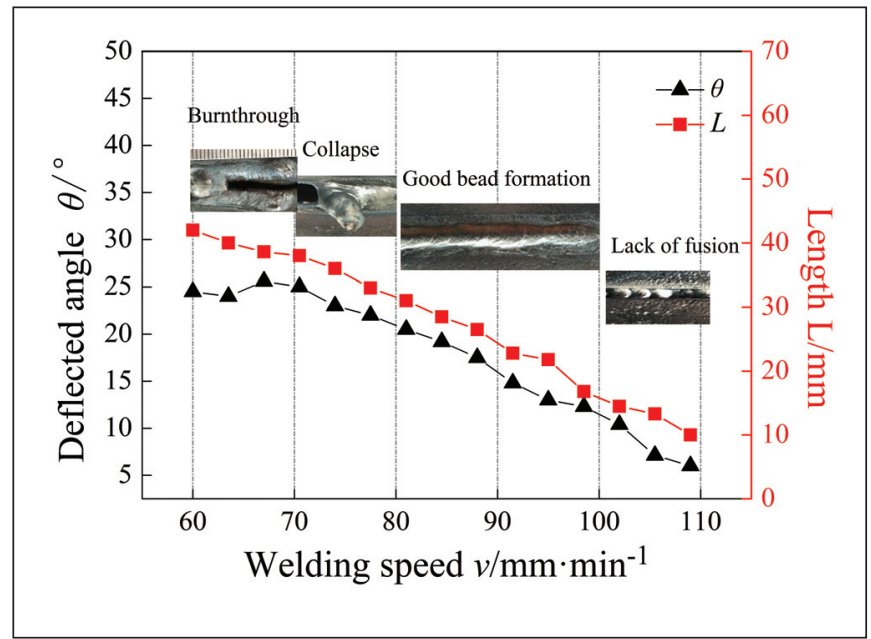

Fig. 7-Statistical curve of correlation. (Pulse frequency $1.5 \mathrm{~Hz}$.)

the internal weld quality of the root pass was detected by the $\mathrm{x}$-ray image as illustrated in Fig. 8C. Consequently, a credible root pass welded bead without defects were obtained in the joining of the medium-thick steel plates using the proposed method. More importantly, these results show the feasibility of using the length and deflected angle of the arc tail flame to identify the weld joint penetration.

\section{Weld Pool Behavior Dependent-Verification of the Monitoring Feasibility}

Previous investigations reported that the dynamic change of the weld pool, such as fluid flow pattern, surface deformation, and solidifying rate strongly influenced the weld bead formation and its properties (Refs. 42-44). Moreover, real-time observation of the weld pool variations in this work indicated that the surface deformation interacted closely with the keyhole evolution, which in turn changed the transformation of the weld penetration status in the entire welding process as well as the ultimate weld bead quality. Thereby, the morphologies of the weld pool in the lowfrequency pulsed GTA root pass welding were captured to further demonstrate the monitoring feasibility of the proposed method. The effects on the weld joint penetration were thoroughly analyzed using an established model integrating the weld bead formation - Fig. 9 . 


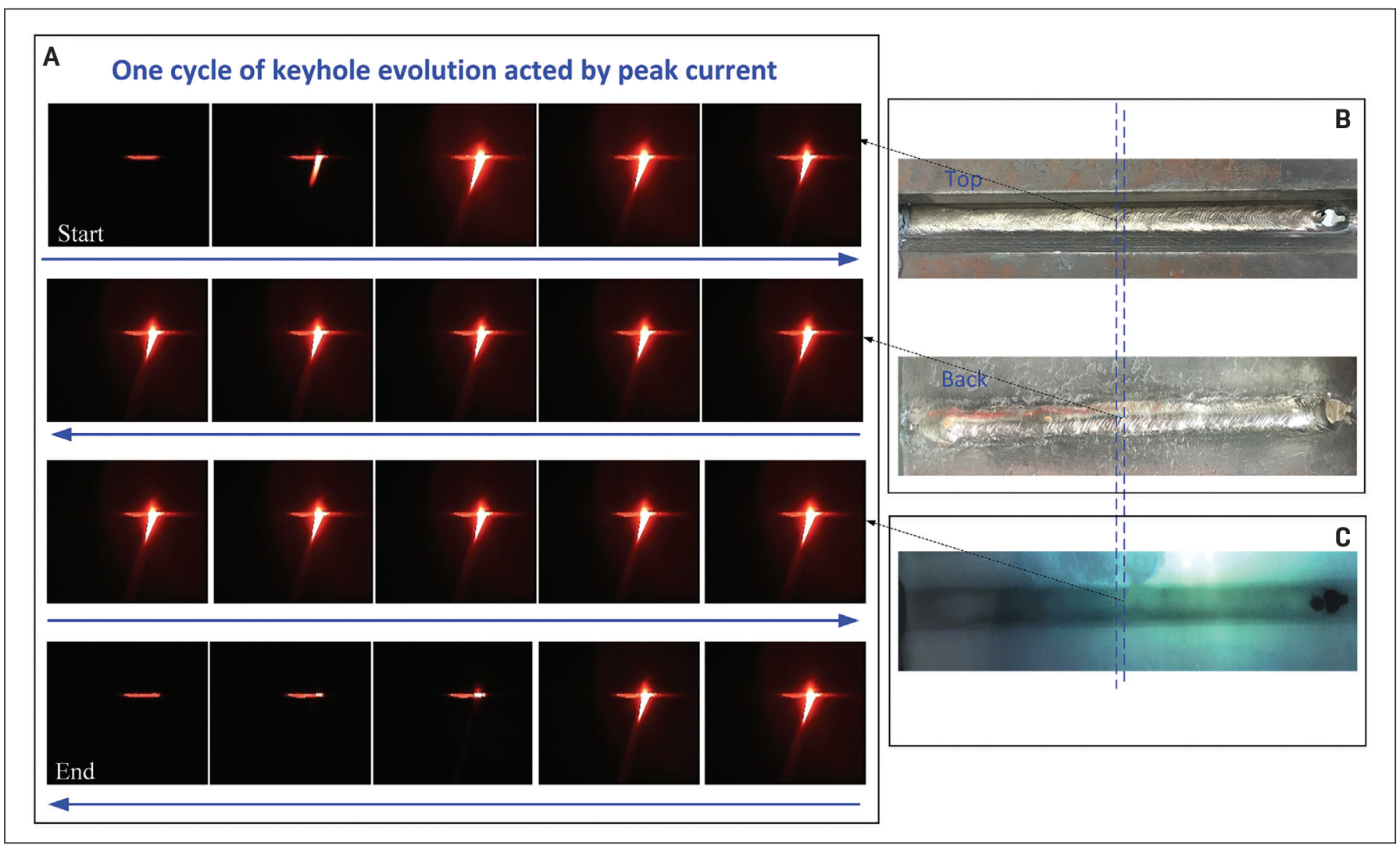

Fig. 8 - In stable root pass welding process: A - Arc tail flame; B - weld bead shape; $C$ - x-ray image.

Figure 9A shows the top morphologies of the weld pool captured at the peak and base current period as well as the distribution of all forces that acted on the weld pool, including the arc pressure $F_{e}$, gas pressure $F_{b}$, surface tension $F_{\delta}$, and gravity $F_{g}$. Figure $9 \mathrm{~B}$ depicts the weld bead shape formed with the different welding parameters. When the peak current acted on the weld pool, the weld pool surface quickly deformed and collapsed in the center area due to the large plasma arc pressure $F_{e}$, thus squeezing out the molten metal to the back of the weld pool in the root pass. Simultaneously, the melted metal in the root pass was forcefully pushed from the center to the sides of the root face due to the strong compressed arc pressure (Fig. 9A, left-hand images), such that the bottom metal of the weld pool was directly fused by the compressed arc. Moreover, the melted bottom metal was intensively flowed by the surface tension

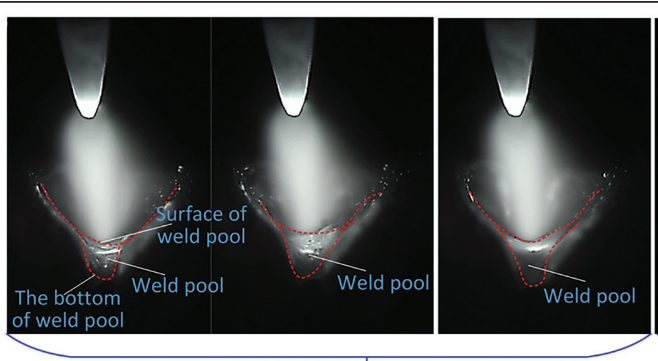

Peak current period

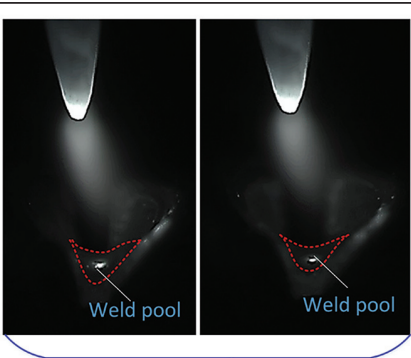

Base current period
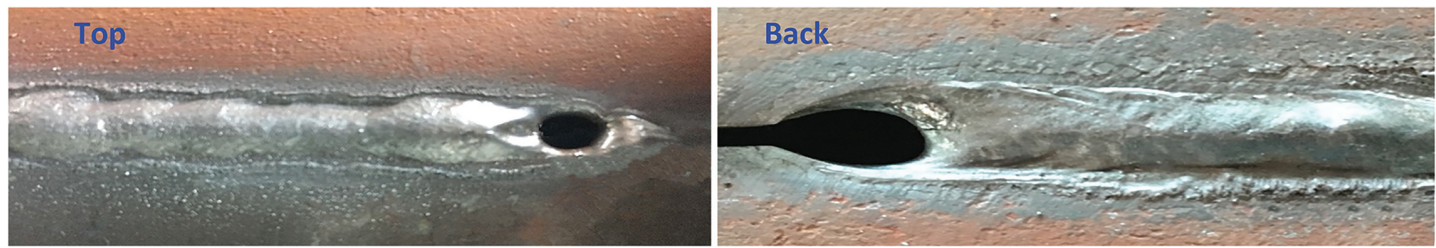

Fig. 9-Images of weld pool and weld bead shape. A - Morphology and established model of a weld pool; B - weld bead shape. 
and the electromagnetic force, and the thermal convection was finally formed. As a result, the root face was completely fused, and a keyhole was generated synchronously. If the thermal-force coupled balance was dynamically guaranteed by adjusting the welding parameters in real time, the keyhole would be evolved steadily. Consequently, the dimension of the keyhole would be changed modestly, such that the length and deflected angle of the arc tail flame would vary in a small range scale.

The above analysis of the weld pool dynamic behavior verified that the stable evolution of the keyhole and its necessity in obtaining sound one-sided welding with back-formation bead. Hence, the results demonstrated the feasibility of using the length and deflected angle of the arc tail flame to estimate the weld joint penetration in the root pass welding, which may provide a new way to control the weld penetration in a groove condition to automatically weld the medium-thick steel plates.

\section{Conclusion}

Based on this study, the following conclusions were drawn.

1. Medium-thick steel plates with a 5-mm root face and 2-mm root opening were reliably joined by the low-frequency and large duty-cycle pulsed GTAW. Sound one-sided welding with back-formation bead was obtained steadily.

2. The length and deflected angle of the arc tail flame were negatively correlated with the welding speed and positively correlated with the weld joint penetration. A highquality weld bead was achieved when the length and deflected angle range from $15 \mathrm{deg}, 20 \mathrm{~mm}$ to $19 \mathrm{deg}, 27 \mathrm{~mm}$, respectively.

3. The results demonstrated the feasibility of using the length and deflected angle of the arc tail flame to estimate the weld joint penetration in the root pass welding, which may provide a new way to control the weld joint penetration in the medium-thick steel plate root pass welding condition.

4. The experimental and weld pool dynamic-dependent analyses demonstrated the feasibility of this proposed method for monitoring the weld joint penetration in the groove welding condition.

\section{Acknowledgments}

This work is funded by the National Natural Science Foundation of China (\#51905245), Gansu Natural Science Foundation (\#20JR5RA455), Special Fund of Guiding Scientific and Technological Innovation and Development of Gansu Province (\#2019ZX-08), Hong Liu Outstanding Talent Training Plan of Lanzhou University of Technology, the Open Fund of Opto-Mechatronic Equipment Technology Beijing Area Major Laboratory, and Key Research Project of Gansu (\#20YF8GA054).

\section{References}

1. Pandey, C., Mahapatra, M. M., Kumar, P., and Saini, N. 2018. Autogenous tungsten inert gas and gas tungsten arc with filler welding of dissimilar P91 and P92 steels. Journal of Pressure Vessel Technology-Transactions ASME 140(2): 591-596. DOI: 10.1115/ 1.4039127

2. Pandey, C., Narang, H. K., and Saini, N. 2017. Microstructure and transverse shrinkage stress analysis in GTA welds of P91 steel pipe. International Journal of Steel Structures 17: 763-774. DOI: 10.1007/s13296-017-6030-8

3. Adamantiades, A., and Kessides, I. 2009. Nuclear power for sustainable development: Current status and future prospects. Energy Policy 37(12): 5149-5166.

4. Beer, D. 2018. Guidebook for the Design of ASME Section IX Pressure Vessels.

5. Brian, L. J. 2001. Keyhole gas tungsten arc welding: A new process variant. $\mathrm{PhD}$ diss., University of Wollongong.

6. Jarvis, B. L., and Ahmed, N. U. 2000. Development of keyhole mode gas tungsten arc welding process. Science and Technology of Welding and Joining 5(1): 1-7. DOI:10.1179/136217100322910624

7. Lathabai, S., Jarvis, B. L., and Barton, K. J. 2001. Comparison of keyhole and conventional gas tungsten arc welds in commercially pure titanium. Materials Science and Engineer: A Structure Material 299(1-2): 81-93. DOI: 10.1016/ S0921-5093(00)01408-8

8. Feng, Y., Luo, Z., Liu, Z., Li, Y., Luo, Y., and Huang, Y. 2015. Keyhole gas tungsten arc welding of AISI 316L stainless steel. Materials \& Design 85: 24-31. DOI: 10.1016/j.matdes.2015.07.011

9. Liu, Z., Fang, Y., Cui, S., Luo, Z., Liu, W., Liu, Z., Jiang, Q., and Yi, S. 2016. Stable keyhole welding process with K-TIG. Journal of Materials Processing Technology 238: 65-72. DOI: 10.1016/ j.jmatprotec.2016.07.005

10. Liu, Z. M., Fang, Y. X., Qiu, J. Y., Feng, M. N., and Yuan, J. R. 2017. Stabilization of weld pool through jet flow argon gas backing in C-Mn steel keyhole TIG welding. Journal of Materials Processing Technology 250: 132-143. DOI: 10.1016/j.jmatprotec.2017.07.008

11. Kovacevic, R., Zhang, Y. M., and Li, L. 1996. Monitoring of weld joint penetration based on weld pool geometrical appearance. Welding Journal 75(10): 317-s to 329-s.

12. Chen, S. B., Zhao, D. B., Lou, Y. J., and Wu, L. 2004. Computer vision sensing and intelligent control of welding pool dynamics. Robot Weld Intelligent Automation LNCIS 229: 25-55. DOI: 978-3540-20804-4

13. Ario, S., Baskoro, R. M., and Masashi, K. 2009. Welding penetration control for aluminium pipe welding using omni-directional vision-based monitoring of molten pool. Jointing Conference collected papers 27(2): 17-21. DOI: 10.2207/qjjws.27.17s

14. Huang, W., and Kovacevic, R. 2012. Development of a realtime laser-based machine vision system to monitor and control welding processes. International Journal of Advanced Manufacturing Technology 63(1-4): 235-248. DOI: 10.1007/s00170-012-3902-0

15. Guu, A. C., and Rokhlin, S. I. 1989. Computerized radiographic weld penetration control with feedback on weld pool depression. Materials Evaluation 47: 10-14. DOI: 10.1016/09638695(96)84896-1

16. Beardsley, H., Zhang, Y. M., and Kovacevic, R. 1994. Infrared sensing of full penetration state in gas tungsten arc welding. International Journal of Machine Tools and Manufacture 34(8): 1079-1090. DOI: 10.1016/0890-6955(94)90014-0

17. Chen, W. H., and Chin, B. A. 1990. Monitoring joint penetration using infrared sensing techniques. Welding Journal 69(4): 181 -s to 185 -s.

18. Vasudevan, M., Chandrasekhar, N., Maduraimuthu, V., Bhaduri, A. K., and Raj, B. 2011. Real-time monitoring of weld pool during GTAW using infra-red thermography and analysis of infrared thermal images. Welding in the World 55(7): 83-89. DOI: 10.1007/BF03321311

19. Siores, E. 1988. Development of a real-time ultrasonic sensing system for automated and robotic welding. $\mathrm{PhD}$ diss., Brunel University.

20. Carlson, N. M., and Johnson, J. A. 1988. Ultrasonic sensing of weld pool penetration. Welding Journal 67(11): 239-s to 246-s. 
21. Mizota, H., Nagashima, Y., and Obana, T. 2015. Fundamental study of molten pool depth measurement method using an ultrasonic phased array system. Japanese Journal of Applied Physics 54(7S1): 07HC03-1-4. DOI: 10.7567/JJAP.54.07HC03

22. Shilov, A. V., Kushner, A. V., and Novikov, V. A. 2016. The detection of real defects in ferromagnetic objects using a magneticfield imaging film. Russian Journal of Non-destructive Testing 52(4): 220-225. DOI: 10.1134/S1061830916040070

23. Pal, K., and Pal, S. K. 2011. Monitoring of weld penetration using arc acoustics. Materials and Manufacturing Processes 26: 684-693. DOI: 10.1080/10426910903496813

24. Lv, Na., Xu, Y. L., Zhong, J. Y., Chen, H. B., Wang, J. F., and Chen, S. B. 2012. Research on detection of welding penetration state during robotic GTAW process based on audible arc sound. Industrial Robot: An International Journal 40(5): 474-493. DOI: 10.1108/IR-09-2012-417

25. Aendenroomer, A. J. R., and Ouden, G. D. 1998. Weld pool oscillation as a tool for penetration sensing during pulsed GTA welding. Welding Journal 77(5): 181-s to 187-s.

26. Ju, J. B., Hiroyuki, H., and Suga, A. 2004. Oscillation of molten pool by pulsed assist gas oscillating method and penetration control using peculiar frequency. Journal of High Temperature Society 30(5): 263-269. DOI: 10.7791/jhts.30.263

27. Wang, Q. L., and Lin, S. B. 1988. Detection of the weld full penetration signals of a traveling arc in GTAW process. Processing of JIW Asian Pacific Regional Welding Congress, Hobart, Australia.

28. Wang, Q., Geng, Z., and Yin, S. 1991. Real-time full penetration control with sensor in the TIG welding of aluminum alloy. Proceedings of International Conference Joining 9-16.

29. Chen, Z., Chen, J., and Feng, Z. 2019. 3D Weld pool surface geometry measurement with adaptive passive vision images. Welding Journal 98(12): 379-s to 386-s. DOI: 10.29391/2019.98.031

30. Chen, Z., Chen, J., and Feng, Z. 2018. Welding penetration prediction with passive vision system. Journal of Manufacturing Processes 36: 224-230. DOI: 10.1016/j.jmapro.2018.10.009

31. Chandrasekhar, N., Vasudevan, M., Bhaduri, A. K., and Jayakumar, T. 2015. Intelligent modeling for estimating weld bead width and depth of penetration from infra-red thermal images of the weld pool. Journal of Intelligent Manufacturing 26(1): 59-71. DOI: $10.1007 /$ s10845-013-0762-x

32. Zhang, S. Q., Hu, S. S., and Wang, Z. J. 2016. Weld penetration sensing in pulsed gas tungsten arc welding based on arc voltage. Journal of Materials Processing Technology 229: 520-527. DOI: 10.1016/ j.jmatprotec.2015.09.034

33. Li, X. R., Shao, Z., Zhang, Y. M., and Kvidahl, L. 2013. Monitoring and control of penetration in GTAW and pipe welding. Welding Journal 92(6): 190-s to 196-s.

34. Aendenroomer, A. J. R., and Ouden, G. D. 1998. Weld pool oscillation as a tool for penetration sensing during pulsed GTA welding.
Welding Journal 77(5): 181-s to 187-s.

35. Li, C., Wang, Q., Jiao, W., Johnson, M., et al. 2020. Deep learning-based detection of penetration from weld pool reflection images. Welding Journal 99(9): 239-s to 245-s. DOI: 10.29391/2020.99.022

36. Chen, J. S., Chen, J., Zhang, K., et al. 2018. Dynamic reflection behaviors of weld pool surface in pulsed GTAW. Welding Journal 97(6): 191- s to 153-s. DOI: 10.29391/2018.97.017

37. Zhang, K., Zhang, Y. M., Chen, J. S., et al. 2017. Observation and analysis of three-dimensional weld pool oscillation dynamic behaviors. Welding Journal 96(5): 143-s to 206-s.

38. Shi, Y., Li, C. K., and Du, L. M. 2016. Frequency characteristics of weld pool oscillation in pulsed gas tungsten arc welding. Journal of Manufacturing Processes 24: 145-151. DOI: 10.1016/j.jmapro.2016. 08.010

39. Li, C. K., Shi, Y., and Gu, Y. F. 2018. Monitoring weld pool oscillation using reflected laser pattern in gas tungsten arc welding. Journal of Materials Processing Technology 255: 876-885. DOI: 10.1016/j.jmatprotec.2018.01.037

40. Shi, Y., Zhang, G., Ma, X. J., et al. 2015. Laser-vision-based measurement and analysis of weld pool oscillation frequency in GTAW-P. Welding Journal 94(5): 176-s to 187-s.

41. Meng, X. M., and Qin, G. L. 2019. A theoretical study of molten pool behavior and humping formation in full penetration high-speed gas tungsten arc welding. International Journal of Heat and Mass Transfer 132: 143-153. DOI:10.1016/j.ijheatmasstransfer. 2018.12.004

42. Zhao, M., Chen, J. X., Yu, M. B., et al. 2020. Effect of transverse magnetic field on weld formation and microstructure and properties of high-speed hot-wire tungsten inert gas welding joints. Science and Technology of Welding and Joining. DOI: 10.1080/13621718. 2020.1730546

43. Huang, L. J., Hua, X. M., and Wu, D. S. 2018. Relationship between the weld pool convection and metallurgical and mechanical properties in hybrid welding for butt joint of 10-mm-thick aluminium alloy plate. Welding in the World 62(5): 895-903. DOI: 10.1007/ s40194-018-0591-9

44. Wu, C. S., Chen, J., and Zhang, Y. M. 2007. Numerical analysis of both front- and back-side deformation of fully-penetrated GTAW weld pool surfaces. Computational Materials Science 39(3): 635-642. DOI: 10.1016/j.commatsci. 2006.08.018
ZHENGWEN ZHU, GANG ZHANG (zhanggang@lut.edu.cn), KAIFEI WANG, YU SHI, and MING ZHU are with State Key Laboratory of Advanced Processing and Recycling of Nonferrous Metals, Lanzhou University of Technology, Lanzhou, China. ZHANG is also with Opto-Mechatronic Equipment Technology Beijing Area Major Laboratory, Beijing Institute of Petrochemical Technology, Beijing, China.

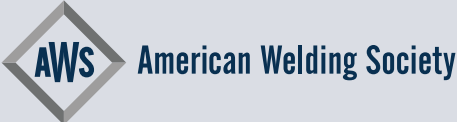

\section{Authors: Submit Research Papers Online}

Peer review of research papers is now managed through an online system using Editorial Manager software. Papers can be submitted into the system directly from the Welding Journal page on the American Welding Society (AWS) website (aws.org) by clicking on "submit papers." You can also access the new site directly at editorialmanager.com/wj/. Follow the instructions to register or log in. This online system streamlines the review process and makes it easier to submit papers and track their progress. By publishing in the Welding Journal, more than 70,000 members will receive the results of your research.

Additionally, your full paper is posted on the AWS website for FREE access around the globe. There are no page charges, and articles are published in full color. By far, the most people, at the least cost, will recognize your research when you publish in the world-respected Welding Journal. 